\title{
Early versus late tracheostomy in cardiovascular intensive care patients
}

\author{
Wilfredo Puentes ${ }^{1}$, Angela Jerath ${ }^{1,2}$, George Djaiani ${ }^{1,2}$, \\ Rosa Maria Cabrerizo Sánchez ${ }^{1}$, Marcin Wąsowicz ${ }^{1,2}$ \\ ${ }^{1}$ Department of Anesthesia and Pain Management, \\ Toronto General Hospital/University Health Network, Canada \\ ${ }^{2}$ Department of Anesthesia, Faculty of Medicine, University of Toronto, Canada
}

\begin{abstract}
Background: Benefits of tracheostomy have been well established. Most of the literature, refers these benefits to general intensive care population, excluding cardiac surgery or including only small number of these patients. On the other hand, there is no clear definition describing the proper time to perform the procedure and defining what are potential benefits of early compared to late tracheostomy. This retrospective cohort aims to assess the potential benefits of early tracheostomy on post-operative outcomes, length of stay and post-tracheostomy complications within cardiac surgical population.
\end{abstract}

Methods: After obtaining REB approval, we conducted a retrospective chart review in a single, tertiary care institution, identifying patients who underwent tracheostomy after cardiac surgery from 1999 to 2006. Time-to-tracheostomy was defined as "early" if $<7$ days or "late" if $\geq 7$ days post-cardiac surgery).

Results: 14,101 patients underwent cardiac surgery over the 7-year study period; from those, 147 (1.36\%) received tracheostomy. 32 (22\%) patients underwent early tracheostomy and 115 (78\%) late tracheostomy. Incidence of atrial fibrillation (31.2\% vs 61.7\%; $P=0.003$ ), kidney dysfunction ( $6.3 \%$ vs $27.2 \% ; P=0.015)$ and kidney failure $18.8 \%$ vs $43.5 \%$; $P=0.013$ ) were lower in the early tracheostomy group. There were no differences on post tracheostomy infection or presence of acute respiratory distress syndrome. Both the ICU and hospital length of stay were significantly shorter in early tracheostomy group, 21.5 (ET) vs 36.9 (LT) days and 37.5 (ET) vs 57.6 (LT) days respectively. There were no differences in mortality between groups.

Conclusions: There are significant benefits in reduction of postoperative morbidities with overall shorter ICU and hospital stay. These benefits may promote faster patient rehabilitation with reduced healthcare costs.

Key words: cardiac surgery, intensive care, tracheostomy, prolonged mechanical ventilation

Most of patients treated within intensive care units (ICU) require temporary airway control to enable mechanical ventilation (MV); once clinical condition is stabilized, the endotracheal tube is removed. For those who require prolonged MV, endotracheal tube is frequently replaced by a tracheostomy tube aiming to facilitate the liberation from $M V$ and to improve patient's comfort; therefore, tracheosto$\mathrm{my}$ is one the most commonly performed procedures in ICU.

About $10 \%$ of patients who require MV for longer than 10 days are tracheostomized, however there is significant variability among institutions and attending ICU physicians [1]. Potential advantages of tracheostomy over orotracheal intubation include: greater patient comfort with secure airway that facilitates tracheal toilet and rapid reduction or discontinuation of sedatives which improves patient communication and decrease the incidence of the delirium, improves mobility and haemodynamic stability with possibly fewer ventilator associated complications. Complications related to tracheostomy include bleeding, hypoxia, oesophageal injury, tracheal stenosis, tracheal granulomas and death. 
Numerous studies undertaken in mixed medical, surgical and trauma intensive care (medico-surgical ICU-MSICU) have shown that early tracheostomy (ET) can reduce patient length of stay, duration of ventilation and sedatives use, which has large cost implications [1-3]. There is no consensus regarding optimal timing to perform tracheostomy, thus there is no clear definition of ET. For example, the American 2001 consensus document advocates the use of early tracheostomy in patients who require prolonged ventilatory assistance but makes no recommendations regarding timing of the procedure [4].

When compared to MSICU, cardiovascular surgical intensive care units (CVICU) face a much more rapid patient turnover whereas overall patient mortality remains lower. On the other hand, increasing age and complexity of cardiac surgical patients, frequently lead to prolonged MV. Depending on institution and definition of prolonged mechanical ventilation it can be applied in up to $20 \%$ of patients. Survival may not be the only outcome of interest in this group as prolonged mechanical ventilated patients often have a high burden of underlying co-morbidities, and prolonged critical illness leaves them vulnerable to recurring episodes of acute complications with a need for subsequent hospital readmissions [5]. Current trends in cardiac surgical practice have demonstrated that the number of coronary artery bypass graft cases have reached plateau and modern tertiary referral centres are operating upon elderly population with a greater burden of co-morbidities, "redo-do" cardiac cases, which will additionally impact on prolonged ICU stay.

There are conflicting data from isolated CVICU environment assessing whether this patient population would have benefits from ET. Additionally, performing ET in postcardiac surgical patients has been marred with concerns over greater risk of deep sternal wound infections given the close proximity of the surgical and tracheostomy sites [6, 7].

This retrospective cohort aims to assess the potential benefits of ET on length of stay; post-operative outcomes and post-tracheostomy complications within cardiac surgical population in single, tertiary care institution.

\section{METHODS}

After the Institutional Review Board approval, we conducted a retrospective chart review to identify patients who underwent tracheostomy after cardiac surgery from 1999 to 2006.

The decision to perform tracheostomy was made by the attending intensivist and cardiac surgeon. There was no formal protocol to proceed with tracheostomy and decision was based on clinical criteria including inability to wean of MV, due to multi-organ dysfunction or failure, neurological injury and severe hyperactive delirium. Tracheostomy was performed either at bedside as a percutaneous technique or as an open procedure within the operating room. Trending from open to percutaneous tracheostomy was also analysed over the time. Post-tracheostomy insertion, patients were weaned of MV with stepwise reduction of pressure support ventilation and trach-mask trials (spontaneous breathing trial). Patients were discharged from ICU after a mandatory period of 48 hours of unsupported ventilation, minimal secretions and adequate gas exchange.

Patients were classified into two groups according timing of tracheostomy; early tracheostomy (performed $<7$ days post cardiac-surgery) and late tracheostomy (LT) (performed $\geq 7$ days post-cardiac surgery). Both groups were compared with respect to patient demographics, surgical characteristics, outcomes and postoperative complications.

\section{STATISTICAL ANALYSIS}

Statistical analysis was conducted with the use of IBM SPSS Statistics computer software (IBM Corporation). Demographic and clinical variables are described as mean \pm SD for normally distributed continuous data, median [IQR] for skewed data and frequencies (\%) for categorical variables. Rate of complications, chest tube drainage, and length of stay were compared using non-parametric Wilcoxon-Rank test for the continuous data and Fisher's exact test for categorical variables. $P<0.05$ was considered statistically significant.

\section{RESULTS}

Tracheostomy was performed in 147 (1.36\%) of a total of 14,101 patients undergoing cardiac surgical procedures over the 7-year study period. Among them, 32 (22\%) patients underwent ET and 115 (78\%) LT. The median intubation time for early and late tracheostomy groups were 7 (5-7) and 13 (10-19) days respectively, $P<0.0001$ (Fig. 1).

Demographic variables and surgical characteristics are presented in Table 1. Postoperative morbidity and mortality are reflected in Table 2 . ET was associated with reduced rates of atrial fibrillation (31.2\% vs $61.7 \% ; P=0.003)$, and lower incidence of kidney dysfunction (6.3\% vs $27.2 \%$; $P=$ 0.015 ) and kidney failure $18.8 \%$ vs $43.5 \% ; P=0.013$ ). There were no differences on post tracheostomy infection or presence of acute respiratory distress syndrome (ARDS). The incidence of sternal wound infection was similar in both groups. Both the ICU and hospital length of stay were significantly shorter in early tracheostomy group, 21.5 (ET) vs 36.9 (LT) days and 37.5 (ET) vs 57.6 (LT) days respectively (Fig. 2). Overall mortality was similar between the two groups. A complete description of postoperative complications is presented in Table 2 .

The percutaneous approach was more commonly used within the ET group, i.e., 25 (78\%) patients versus 67 (58\%) patients in the LT group, $P=0.04$. Figure 3 outlines the pattern of tracheostomy approach change over the 7-year period in favour of percutaneous tracheostomy. 


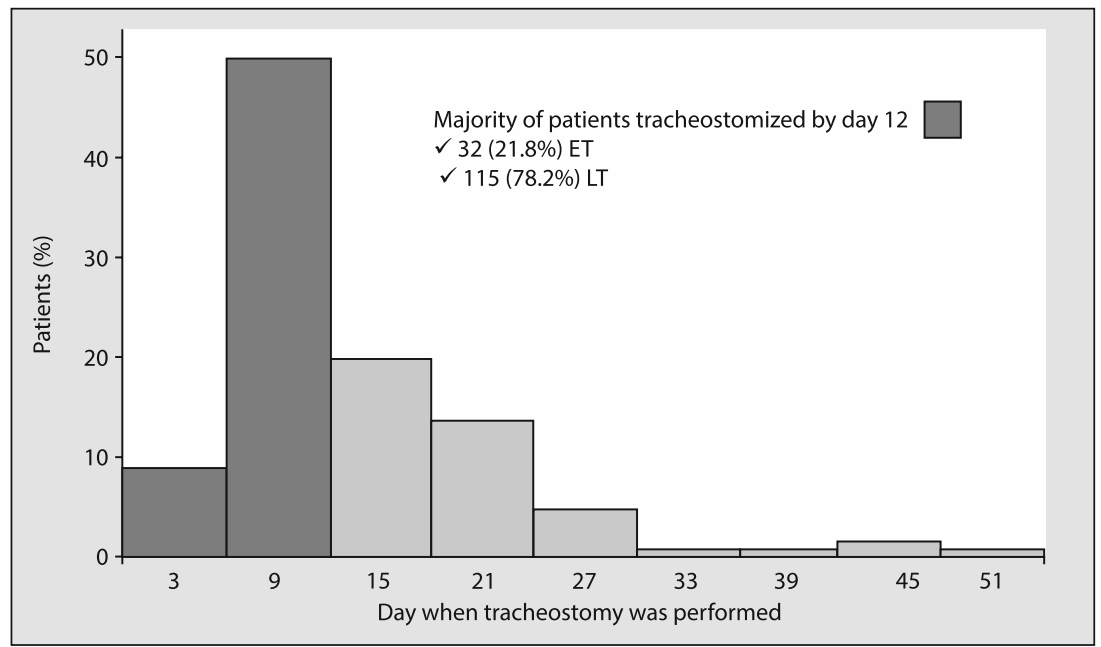

Figure 1. Days of tracheostomy - most of the patients had the tracheostomy performed by the day number 12. 32 (21.8\%) patients correspond to early tracheostomy group (ET) and 115 (78.2\%) to late tracheostomy (LT) group

Table 1. Demographic distribution and intraoperative variables

\begin{tabular}{lccc}
\hline Variables & ET $(\mathrm{n}=32)$ & $\mathrm{LT}(\mathrm{n}=115)$ & $P$ value \\
\hline Age, years & $63.1 \pm 14.0$ & $66.6 \pm 13.2$ & 0.108 \\
BMI, kg m ${ }^{-2}$ & $28.3 \pm 7.7$ & $28.1 \pm 5.8$ & 0.642 \\
Male, $\mathrm{n}(\%)$ & $25(78.1)$ & $73(63.5)$ & 0.141 \\
Hypertension, $\mathrm{n}(\%)$ & $19(59.4)$ & $70(60.9)$ & 0.878 \\
Myocardial Infarction, $\mathrm{n}(\%)$ & $15(46.9)$ & $46(40)$ & 0.545 \\
CCF, $\mathrm{n}(\%)$ & $15(46.9)$ & $51(44.3)$ & \\
LV grade 4 & $2(8)$ & $11(11.5)$ & \\
COPD, $\mathrm{n}(\%)$ & $7(21.9)$ & $19(16.5)$ & 0.600 \\
Preop creatinine, $\mathrm{mmol}^{-1}$ & $137.7 \pm 100$ & $118.2 \pm 69.5$ & 0.198 \\
CVA/TIA, $\mathrm{n}(\%)$ & $8(25)$ & $19(16.5)$ & 0.305 \\
Diabetes, $\mathrm{n}(\%)$ & $11(34.4)$ & $40(35.1)$ & 1 \\
CPB, minutes & $128.2 \pm 47.4$ & $151.9 \pm 67.2$ & 0.065 \\
Elective, $\mathrm{n}$ (\%) & $11(36.7)$ & $39(35.1)$ & \\
CABG, $\mathrm{n}(\%)$ & $10(32.3)$ & $40(35.1)$ & \\
Single Valve, $\mathrm{n}(\%)$ & $3(9.7)$ & $5(4.4)$ & \\
Complex, $\mathrm{n}(\%)^{*}$ & $18(58.1)$ & $69(60.5)$ & \\
Chest tube Loss 24 hr, $\mathrm{mL}$ & $877.5 \pm 770.1$ & $1076.1 \pm 958.6$ & 0.096 \\
ICU creatinine, mmol L-1 & $132.8 \pm 86.4$ & $130 \pm 88.1$ & 0.841 \\
\hline
\end{tabular}

ET — early tracheostomy; LT — late tracheostomy; BMI - body mass index; CVA - cerebro vascular accident; TIA - transient ischemic accident; CCF congestive cardiac failure; COPD - chronic obstructive pulmonary disease; CPB - cardiopulmonary bypass time; $C A B G$ - coronary artery bypass grafting ICU - intensive care unit; ${ }^{*}$ Complex surgery - combination, aortic, redo procedures, $P$ value 0.52 for all procedures

\section{DISCUSSION}

The current study identified that ET performed in cardiac surgical patients was associated with reduced rates of postoperative atrial fibrillation, and lower incidence of renal dysfunction and renal failure. Furthermore, patients undergoing ET had reduced ICU and hospital length of
Table 2. Postoperative complications

\begin{tabular}{|c|c|c|c|}
\hline Variable & ET & LT & $P$ value \\
\hline Atrial Fibrillation, $\mathrm{n}(\%)^{*}$ & $10(31.2)$ & $71(61.7)$ & 0.003 \\
\hline Bleeding, $\mathrm{n}(\%)^{*}$ & $6(18.9)$ & $37(32.2)$ & 0.188 \\
\hline Low cardiac index, $n(\%)^{*}$ & $11(34.4)$ & $39(33.9)$ & 1.0 \\
\hline Renal Dysfunction, $\mathbf{n}(\%)^{*}$ & $2(6.3)$ & $31(27.2)$ & 0.015 \\
\hline Renal Failure, $\mathbf{n}(\%)^{*}$ & $6(18.8)$ & $50(43.5)$ & 0.013 \\
\hline Infection, $\mathrm{n}(\%)^{*}$ & $16(50)$ & $75(65.2)$ & 0.150 \\
\hline Acute Lung Injury, n (\%)* & $1(3.1)$ & $2(1.7)$ & 0.524 \\
\hline ARDS, n (\%)* & $3(9.4)$ & $26(22.6)$ & 0.132 \\
\hline Pre Trach fiO ${ }_{2}$ & $0.5 \pm 0.2$ & $0.5 \pm 0.6$ & 0.252 \\
\hline Pre Trach PEEP, $\mathrm{cm} \mathrm{H}_{2} \mathrm{O}$ & $6.6 \pm 2.5$ & $6.4 \pm 2.3$ & 0.413 \\
\hline Post Trach Infection, $\mathrm{n}(\%)^{* *}$ & $15(46.9)$ & $74(64.3)$ & 0.101 \\
\hline Post Trach Renal Failure, n (\%) & $8(25)$ & $41(35.7)$ & 0.296 \\
\hline Post Trach Mortality, n (\%) & $8(25)$ & $34(29.8)$ & 0.664 \\
\hline
\end{tabular}

stay, however ET did not result in mortality difference. Our findings indicated that timing of tracheostomy was not associated with increased rate of sternal wound infection. Additionally, our results confirmed the trends of changing practice with tracheostomies being increasingly performed at the bedside using a percutaneous approach.

The lower rates of atrial fibrillation and renal failure could be attributed to shorter cardiopulmonary bypass times and lower sedation requirements in the early tracheostomy group. At the same time, less sedation is associated with better hemodynamic performance with lower requirement for vasoactive drugs to reverse druginduced hypotension. 


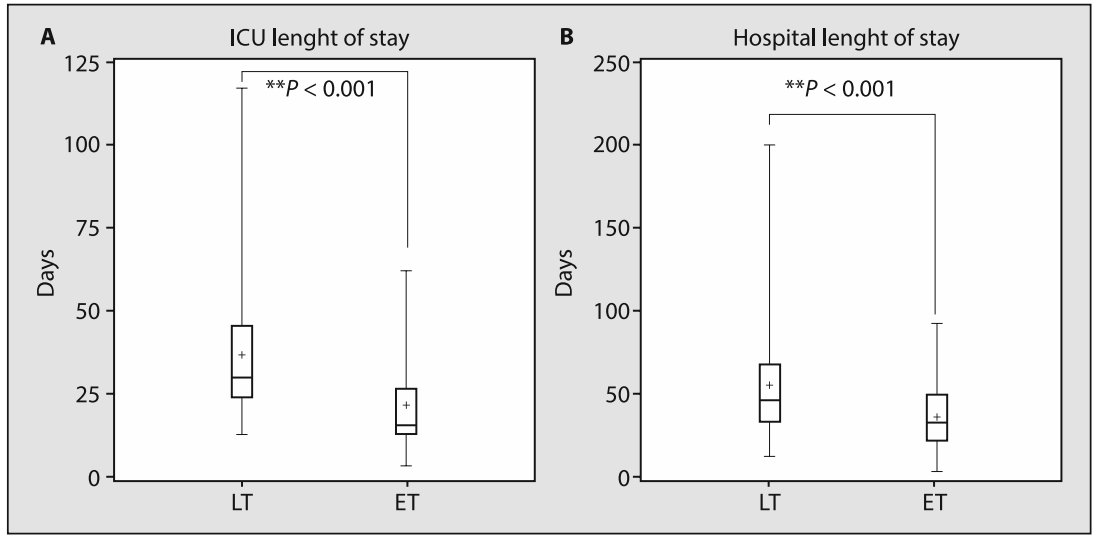

Figure 2. Length of stay - a representation of length of stay in the intensive care unit (ICULOS) (A) and overall in-Hospital time (HLOS) (B). There is a significant difference in favour of early tracheostomy (ET) in both ICULOS and HLOS, 21.5 (ET) vs 36.9 (LT) days and 37.5 (ET) vs 57.6 (LT) days respectively

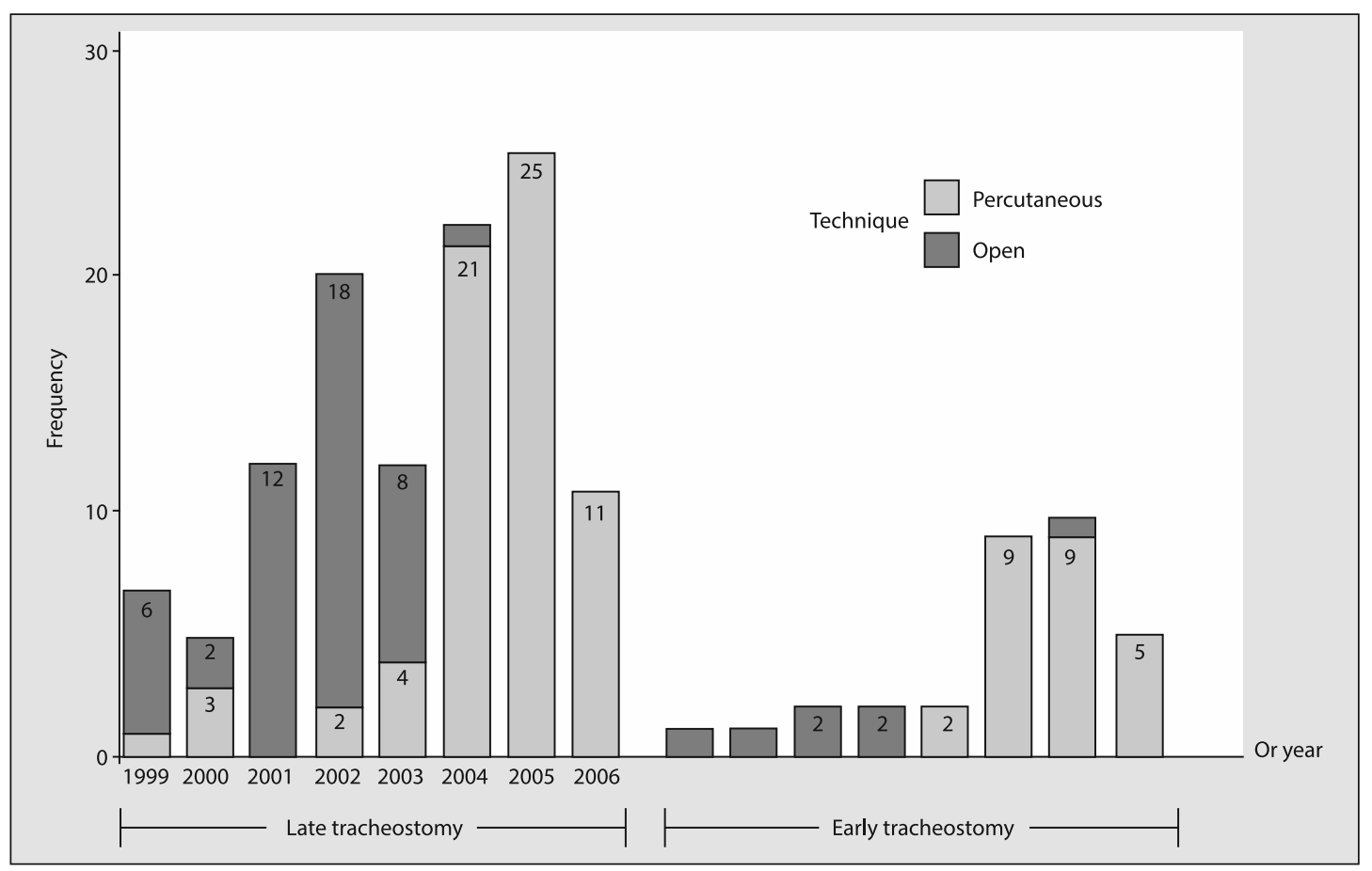

Figure 3. Changing trends from open to percutaneous tracheostomy

Our findings corroborated with recent systematic review of adult ICU patients suggesting that early tracheostomy may reduce duration of ICU length of stay without any reduction in mortality [8]. On the contrary, Rumbak et al. showed a significant reduction in mortality (31.7\% vs $61.7 \%)$ and lower incidence of pneumonia (5\% vs $25 \%$ ) in their randomized controlled trial of ET ( 48 hrs) vs LT (14-16 days) tracheostomy in medical ICU patients. The shortcoming of this study was that patients who received the tracheostomy between days 3 and 13 were not included in the analysis [9]. A larges cohort of 11.000 patients requiring tracheostomy over a period of 12 years was recently studied in province of Ontario. Authors found that each additional day of delaying tracheostomy was associated with increased long-term mortality [10]. Similar results were found by Shaw et al. in the largest retrospective cohort addressing this issue. This group compared ET ( $<7$ days) vs LT ( $>10$ days) excluding those patients whom tracheostomy was performed between days 7 and 10. Total 49,191 patients from 185 different medical centres were included in this analysis; 21,029 patients in the ET cohort and 28.162 in the LT cohort. There were significant differences in incidence of VAP (12\% ET vs $15 \%$ 
LT; $P<0.0001$ ), ICU and hospital length of stay (16 days ET vs 27 days $\mathrm{LT}, P<0.0001)$ and ( 25 days ET vs 38 days LT; $P$ $<0.0001$ ). Mortality was lower in ET group (14\% ET vs $21 \%$ $\mathrm{LT} ; P<0.0001)$. ET was found to be independent predictor of mortality but not incidence of VAP [11].

A recent meta-analysis published for Huang et al. evaluated 9 randomized clinical trials with 2,072 patients [12]. Compared to LT or prolonged MV, they did not find significantly difference of ET in terms of short-term mortality [relative risk $(\mathrm{RR})=0.91 ; 95 \%$ confidence intervals $(\mathrm{cls})=$ $0.81-1.03 ; P=0.14]$ or long-term mortality VAP or duration of MV. This result differs from a recent Cochrane Collaboration review that involved seven randomized controlled trials including 1903 participants where the authors found lower mortality rates in the ET as compared with the LT group (risk ratio $=0.83 ; 95 \% \mathrm{Cl} 0.70$ to $0.98, P=0.03$ ) [13].

Finally, Young et al. published recently the largest randomized study investigating early vs late tracheostomy (The TracMan study) [14]. In this multicentre trial, patients from over 70 ICUs were randomized to early (within 4 days) or LT (after 10 days) when predicted ventilation time was longer than a week. There was no difference in early and late mortality between groups. Additionally, only $45 \%$ of patients assigned to LT received it, the rest of patients improved and ultimately did not require MV. Authors concluded that ET did not improve important clinical outcomes, moreover indicated that ability of clinicians to predict prolonged $\mathrm{MV}$ was poor.

As oppose to general ICU population, there are only few publications investigating the impact of tracheostomy timing in cardiac surgical population. Yavas et al. published a single centre review of 205 patients and found that ET was associated with earlier mobilization, earlier enteral feeding, lower incidence of infections, and lower mortality. Furthermore, this study confirmed that percutaneous tracheostomy performed after the cardiac surgical procedure did not increase the incidence of sternal osteomyelitis and sternal wound dehiscence [15]. Trouillet and colleagues conducted a prospective cohort study of 163 patients who were still mechanically ventilated 3 days after cardiac surgery. They reported that $50(31 \%)$ patients had been successfully weaned, 78 (48\%) were still receiving MV, and 35 (21\%) had died by day 10 . Urine output $500 \mathrm{~mL} 24 \mathrm{~h}^{-1}$ or greater, Glasgow coma score of 15 , arterial bicarbonate $20 \mathrm{mmol} \mathrm{L}^{-1}$ or greater, platelet count $100 \mathrm{~g} \mathrm{~L}^{-1}$ or greater, patients without inotropic support with epinephrine/norepinephrine, and absence of lung injury were predictive of successful weaning from MV by day 10 . Based on their statistical modelling the authors concluded that only $3 \%$ to $17 \%$ of the patients would have received a needless intervention (tracheostomy) [16] Subsequently, the same group of authors conducted a prospective, randomized, single centre trail, which assigned cardiac surgical patients with predicted prolonged MV (more than 7 days) to early (4 days) or LT (15 days). Only $27 \%$ of patients allocated to late group received a tracheostomy. The results revealed no difference in duration of MV, hospital stay and 90-day mortality. VAP as well as incidence of other infections were also similar between the ET and LT group. However, patients within the ET group required less sedation and experienced greater comfort of ICU stay and earlier resumption of patient autonomy $[17,18]$. These findings do not corroborate with those of Devarajan et al., who compared ET ( $<10$ days post-cardiac surgery) to LT (14-28 days post-cardiac surgery) and demonstrated significant benefits of ET. This study used propensity matching of 114 patients and showed ET was associated with lower mortality ( $21.1 \%$ vs $40.4 \%$ ) and cardiac morbidity (14 vs $33 \%$ ). [19]. A retrospective single centre study from Ben-Avi et al. contrasts with our results in terms of mortality. They compared ET (< 14 days post-cardiac surgery) in 90 patients to LT (> 14 post-cardiac surgery days) in 109 patients. The mortality rate at 3 months, 6 months, 1 year, and 2 years was 37\%, $48 \%, 56 \%$, and $58 \%$ in ET group and $58 \%, 70 \%, 74 \%$, and $77 \%$ in LT group, respectively. They also report lower incidence of deep sternal wound infection $(1.11 \%$ ET vs $8.26 \%$ LT), but no difference in ICU and hospital length of stay or days on MV [20]. Hosseinian et al. published a retrospective cohort evaluating the impact of timing of tracheostomy on outcomes of patients with respiratory failure after cardiac surgery [21]. Their study involved 1000 hospitals in 45 states across United States. A total of 2,063,227 patients underwent coronary artery bypass grafting (CABG), valve surgery or both. Patients who underwent closed cardiac valvotomy, percutaneous valvuloplasty, or any pulmonic procedure were excluded. A total of 434,519 patients met criteria and were included in analysis. From those, 400,750 (92.2\%) did not present any respiratory failure, $26,046(6 \%)$ presented respiratory failure but did not require tracheostomy. Finally, 7,723 patients ( $1.8 \%$ ) were managed with tracheostomy. This study highlights several strong independent predictors of respiratory failure: female gender, increasing age (octogenarians), chronic obstructive airway disease, chronic renal insufficiency, and valve surgery. Tracheostomy was performed in $22.9 \%$ of patients with respiratory failure. $13.6 \%$ of tracheostomies were performed before the day 5 and $20.5 \%$ on $21^{\text {st }}$ postoperative day or later. Authors concluded that ET is associated with lower operative mortality than later tracheostomy; $20.5 \%$ days 0 to $5,21.6 \%$ days 9 to $10,25.3 \%$ days 11 to $15,24.9 \%$ days 16 to 20 , and $29.6 \%$ if > 20 days after cardiac surgery). This results are in contrary to our findings and also to the results presented in the TracMan study.

Our study has several important limitations. Its retrospective nature precludes us from drawing any causality between the early tracheostomy and lower postoperative 
morbidity. Furthermore, we did not perform a formal cost analysis but reductions in ICU and hospital LOS is always associated with considerable cost savings and resource optimization. Finally, the number of patients who underwent tracheostomy is quite low when compared to other studies.

In summary, our study has demonstrated that ET ( $<7$ days) can be safely performed with no increased risk of sternal wound infection or dehiscence post cardiac surgery. There are significant benefits in reduction of postoperative morbidities with overall shorter ICU and hospital stays. These benefits ultimately promote faster patient rehabilitation with reduced healthcare costs.

\section{ACKNOWLEDGEMENTS}

1. Source of founding: Department of Anesthesia and Pain Management, Toronto General Hospital.

2. The authors declare no conflict of interest.

\section{References:}

1. Armstrong PA, McCarthy MC, Peoples JB: Reduced use of resources by early tracheostomy in ventilator-dependent patients with blunt trauma. Surgery 1998; 124: 763-766.

2. Combes A, Luyt CE, Nieszkowska A, Trouillet IL, Gibert C, Chastre J: Is tracheostomy associated with better outcomes for patients requiring long-term mechanical ventilation? Crit Care Med 2007; 35: 802-807.

3. Rodriguez JL, Steinberg SM, Luchetti FA, Gibbons KJ, Taheri PA, Flint LM: Early tracheostomy for primary airway management in the surgical critical care setting. Surgery 1990; 108: 655-659.

4. Maclntyre NR, Cook DJ, Ely EW Jr. et al.: Evidence-based guidelines for weaning and discontinuing ventilatory support: a collective task force facilitated by the American College of Chest Physicians; the American Association for Respiratory Care; and the American College of Critical Care Medicine. Chest 2001; 120:375s-95s.

5. MacIntyre NR, Epstein SK, Carson Set al.: Management of patients requiring prolonged mechanical ventilation: report of a NAMDRC consensus conference. Chest 2005; 128: 3937-3954.

6. Rahmanian PB, Adams DH, Castillo JG, Chikwe J, Filsoufi F:Tracheostomy is not a risk factor for deep sternal wound infection after cardiac surgery. Ann Thorac Surg 2007; 84: 1984-1991.

7. Wouters $R$, Wellens F, Vanermen H, De Geest R, Degrieck I, De Meerleer $F$ : Sternitis and mediastinitis after coronary artery bypass grafting. Analysis of risk factors. Tex Heart Inst J 1994; 21: 183-188.

8. Griffiths J, Barber VS, Morgan L, Young JD: Systematic review and metaanalysis of studies of the timing of tracheostomy in adult patients undergoing artificial ventilation. BMJ 2005; 330: 1243.

9. Rumbak MJ, Newton M, Truncale T, Schwartz SW, Adams JW, Hazard $P B$ : A prospective, randomized, study comparing early percutaneous dilational tracheotomy to prolonged translaryngeal intubation (delayed tracheotomy) in critically ill medical patients. Crit Care Med 2004; 32: 1689-1694.

10. Scales DC, Thiruchelvam D, Kiss A, Redelmeier DA: The effect of tracheostomy timing during critical illness on long-term survival. Crit Care Med 2008; 36: 2547-5557. doi: 10.1097/CCM.0b013e31818444a5.

11. Shaw JJ, Santry HP: Who Gets early tracheostomy?: evidence of unequal treatment at 185 academic medical centers. Chest 2015; 148: 1242-1250. doi: 10.1378/chest.15-0576.

12. Huang H, Li Y, Ariani F, Chen $X$, Lin J:Timing of tracheostomy in critically ill patients: a meta-analysis. PloS one 2014; 9: e92981. doi: 10.1371/ journal.pone.0092981.

13. Andriolo $B N$, Andriolo $R B$, Saconato $H$, Atallah $A N$, Valente $O$. Early versus late tracheostomy for critically ill patients. Cochrane Database Syst Rev 2015; 1: CD007271. doi: 10.1002/14651858.CD007271.pub3.

14. Young $D$, Harrison DA, Cuthbertson $B H$, Rowan $K$. Effect of early vs late tracheostomy placement on survival in patients receiving mechanical ventilation: the TracMan randomized trial. JAMA 2013; 309: 2121-2129. doi: 10.1001/jama.2013.5154

15. Yavas S, Yagar S, Mavioglu L et al.:Tracheostomy: how and when should it be done in cardiovascular surgery ICU? J Card Surg 2009; 24: 11-18. doi: 10.1111/j.1540-8191.2008.00695.x.

16. Trouillet $\mathrm{J}$, Combes A, Vaissier Eet al.: Prolonged mechanical ventilation after cardiac surgery: outcome and predictors. J Thorac Cardioveasc Surg 2009; 138: 948-953. doi: 10.1016/j.jtcvs.2009.05.034.

17. Trouillet $J$, Luyt $C E$, Guiguet $M$ et al.: Early percutaneous tracheotomy versus prolonged intubation of mechanically ventilated patients after cardiac surgery: a randomized trial. Ann Intern Med 2011; 154:373-383. doi: 10.7326/0003-4819-154-6-201103150-00002.

18. Patel SB, Kress JP: Early tracheotomy after cardiac surgery: not ready for prime time. Ann Intern Med 2011; 154: 434-435. doi: 10.7326/00034819-154-6-201103150-00011.

19. Devarajan J, Vydyanathan $A, X u M$ et al.: Early tracheostomy is associated with improved outcomes in patients who require prolonged mechanical ventilation after cardiac surgery. J Am Coll Surg 2012; 214: 1008-16.e4. doi: 10.1016/j.jamcollsurg.2012.03.005.

20. Ben-Avi R, Ben-Nun A, Levin S et al.: Tracheostomy after cardiac surgery: timing of tracheostomy as a risk factor for mortality. J Cardiothorac VasC Anesth 2014; 28: 493-496. doi: 10.1053/j.jvca.2013.10.031.

21. Hosseinian L, Chiang Y, Itagaki S, Polanco A, Rhee A, Chikwe J: Earlier versus later tracheostomy in patients with respiratory failure after cardiac surgery in the United States. J Cardiothorac Vasc Anesth 2014; 28: 488-492. doi: 10.1053/j. jvca.2013.07.023.

\section{Corresponding author:}

Marcin Wąsowicz, MD

Department of Anesthesia and Pain Management,

Toronto General Hospital, University of Toronto

200 Elizabeth Street, Toronto, ON - M5G 2C4, Canada

e-mail:marcin.wasowicz@uhn.ca

Received: 24.10.2015

Accepted: 13.02 .2016 